\title{
CONSIDERAZIONI SUI PROCESSI DI ADATTAMENTO ALL'AMBIENTE
}

\author{
di \\ Elio Caranti
}

1. Il problema dell'influenza che le condizioni ambientali esercitano sugli esseri viventi è sempre attuale - e non soltanto in biologia - come dimostra la continua apparizione di opere dedicate all'argomento.

In campo biologico tali studi si sono rivolti particolarmente al tema dell'adattamento funzionale, con risultati di innegabile portata. Non altrettanto soddisfacenti si possono ritenere quelli riguardanti l'adattamento strutturale, in quanto il moderno sviluppo della genetica aveva portato a considerare del tutto priva di fondamento la teoria lamarckiana dell'ereditarietà dei caratteri acquisiti, nonostante le evidenti difficoltà logiche incontrate nel giustificare - pur facendo appello al meccanismo mutazionistico - l'evoluzione della specie.

Un largo processo di revisione di tale atteggiamento sembra oggi in corso, da una parte perchè più frequenti si sono fatti i richiami alle questioni di ordine generale e dall'altra per l'affiorare, attraverso la sperimentazione, di fatti nuovi i quali hanno indotto a giudicare $\mathrm{i}$ fenomeni ereditari molto più complessi di quanto non potevano apparire a prima vista.

Non sembra, quindi, fuor di luogo un rapido esame dei punti fondamentali del problema, alla luce di teorie antiche e recenti e dei più significativi risultati delle ricerche sperimentali postbelliche.

2. Un sistema isolato, se è teoricamente concepibile, non trova effettivo riscontro in natura. Ogni sistema reale appare, infatti, in relazione più o meno stretta con altri sistemi e la sua conservazione dipende, in ogni momento, dal mantenimento di una certa armonia con questi.

Per i complessi sistemi che sono gli organismi viventi, l'armonia con i sistemi esterni va sotto il nome di adattamento all'ambiente od extraorganico, per distinguerlo dallo adattamento intraorganico, cioè dal necessario coordinamento dei vari sistemi costituenti l'organismo.

Queste due forme di adattamento - fra le quali esiste ovviamente un intimo collegamento - rappresentano l'espressione dei delicati processi atti ad assicurare la sopravvivenza dell'organismo nel corso del ciclo vitale e lo svolgimento delle sue funzioni essenziali, di cui concorrono a determinare le modalità.

Per ciò solo i fenomeni di adattamento rivestono una notevole importanza per quanto riguarda le variazioni, di ordine quantitativo e qualitativo, che si verificano per una data specie; secondo certe teorie, anzi, sarebbero alla base della evoluzione delle specie viventi. 
3. Si dice che un organismo è adattato ad un determinato ambiente quando può in esso soddisfare le sue esigenze essenziali per la conservazione, la riproduzione, lo sviluppo; se non lo fosse, non sarebbe in grado di sopravvivere come individuo e di perpetuarsi come specie ${ }^{1}$.

Rileviamo subito che tale definizione appare molto generica; sufficiente, forse, a definire un habitat, non offre invece un metro per valutare lo stato o la capacità di adattamento di un organismo, che è evidentemente una questione di gradi.

Una simile valutazione presenta senza dubbio molte difficoltà, in quanto l'ambiente può avere ripercussioni diverse sulle funzioni essenziali per la vita.

Le condizioni che l'uomo si è oggi assicurato nei paesi civili, ad esempio, hanno influito favorevolmente sulla conservazione degli individui; sotto certi aspetti (così per quanto riguarda la statura) anche lo sviluppo dell'organismo è stato favorito; ma le funzioni di riproduzione ne hanno risentito con ogni evidenza - e non interessa qui discutere $\mathrm{i}$ fattori operanti in tal senso - in maniera negativa.

D'altra parte, la definizione citata esprime il punto di vista della biologia funzionale. Ora, modificazioni funzionali anche rilevanti possono avvenire a prescindere da modificazioni strutturali, immediate o mediate, in quanto il disegno degli organismi viventi non obbedisce certo al criterio umano dell'economicità: di fronte a fattori di perturbazione, il riequilibrio dei singoli sistemi componenti e del sistema nel suo complesso è basato sull'entrata in azione di riserve latenti e meccanismi di compenso, in gran parte caratteristici dei sistemi organici.

Di conseguenza, soltanto sottoponendo un certo numero di esseri appartenenti ad una data specie a tutte le combinazioni ambientali possibili, si può essere in grado di determinare uno o più optima per quanto riguarda la loro conservazione, il loro sviluppo, la loro riproduzione e l'ampiezza delle possibilità di vita, frutto, secondo alcuni, dell'evoluzione.

4. Dopo un simile esperimento, si presenta il problema di capire come si sia potuto arrivare ad una struttura capace di dispiegare una tale attività funzionale.

Giova premettere che da parte di qualche studioso viene sostenuto un concetto allargato di struttura, in modo da includervi e riconoscervi il fatto che nell'organismo vivente non si troverebbe una struttura spaziale ed un'attività (funzione) la quale a quella sia sovrapposta; l'organismo concreto consisterebbe in una struttura spazio-temporale coincidente con l'attività stessa ${ }^{2}$.

Sembra però che, anche accogliendo una tale concezione, il problema posto non venga sostanzialmente a cadere; semmai dovrebbe essere modificata l'enunciazione, nel senso di prevedere non una struttura, ma un insieme di strutture spazio-temporali le quali presentino una maggiore variabilità funzionale rispetto a quella strutturale.

Vediamo ora come viene normalmente impostato il problema sopra indicato.

Punto di partenza è la constatazione che la struttura di un organismo appare partico-

1 E. S. RusselL, The directiveness of organic activities. Trad. it.: Finalità nelle attività organiche. Firenze, 1950, p. 137.

2 J. NeEdham, Ordine e vita. Torino, 1946, p. 19. 
larmente adatta alle funzioni che l'organismo stesso deve espletare; da qui muove la ricerca dei processi mediante $\mathbf{i}$ quali, nel corso dell'evoluzione, si è potuto conseguire un simile risultato.

Senonchè le caratteristiche essenziali della vita sono comuni a tutti gli esseri viventi, dalle specie inferiori di batteri agli animali più elevati.

Tutti i tipi di organismo contengono, infatti, le stesse classi di composti biochimici ed usano i medesimi metodi di biosintesi e di trasferimento di energia; posseggono le stesse caratteristiche di fibre e membrane ed il medesimo limitato numero di aminoacidi; i meccanismi metabolici - di respirazione, di secrezione, di digestione - la contrattilità, l'eccitazione, il sistema di trasmissione genica ecc. sono analoghi.

Tali elementi sembra siano rimasti sostanzialmente immutati nel corso dell'evoluzione, anche se inizialmente attribuiti a tipi di organizzazione molto semplici — in grado tuttavia di adattarsi all'ambiente.

Posto ciò, quale importanza filogenetica è possibile riconoscere all'adattamento?

5. È noto che la teoria lamarckiana assegna un ruolo fondamentale ai meccanismi di adattamento nella evoluzione delle specie viventi, attraverso l'assunto della ereditarietà dei caratteri acquisiti.

Ma una tale forma di ereditarietà, mentre dà modo di giustificare le modificazioni funzionali, non permette di spiegare le diversificazioni strutturali - al di fuori, forse, di alcuni caratteri aventi un rilievo piuttosto marginale, come può essere la colorazione della pelle umana.

Se, infatti, le caratteristiche essenziali per la vita sono le medesime in ogni tipo di organismo, non si vede in quale maniera siano potute emergere forme tanto differenti, assai difficilmente riconducibili ad adattamenti imposti dall'ambiente ${ }^{3}$.

Pure per la teoria darwiniana l'adattamento all'ambiente costituisce un fenomeno della massima importanza, in quanto la direzione e l'intensità della selezione dovrebbero risultare dall'adattabilità degli individui formanti la specie.

Anche qui si osserva che, non rappresentando l'organizzazione fondamentale dei diversi tipi organici il risultato di adattamenti, essa non può avere un valore selettivo ${ }^{4}$.

La selezione, d'altra parte, se concorre indubbiamente a determinare la diffusione di certi caratteri, non è in grado di spiegare la comparsa di nuovi caratteri. Ed il mutazionismo - allorchè venga concepito esclusivamente come un meccanismo casuale non è sufficiente a rendere conto della progressione delle forme viventi che caratterizza l'evoluzione.

6. Ma la tesi che le mutazioni, anche se insorte sotto l'azione di fattori esterni, siano sempre a carattere casuale - sostenuta tuttora dalla maggioranza dei genetisti - ha da tempo dato luogo a contestazioni aventi un valido fondamento logico.

«Dall'osservazione dell'uomo - scriveva molti anni fa un illustre biologo, — si

${ }^{3}$ B. DURKen, Entwicklungsbiologie und Ganzheit. Trad. it.: Biologia dello sviluppo e olismo. Firenze, 1943, p. 222 .

B. Durken, op. cit., pp. 223-24. 
direbbe che il plasma germinativo subisca a poco a poco l'influenza dell'ambiente... Certamente l'individuo non trasmette ai suoi discendenti i caratteri da lui assunti, ma i suoi umori si modificano necessariamente secondo il mondo cosmico, e le sue cellule sessuali si adattano come le altre a questi mutamenti del mezzo interno " 5 .

Il Gini, che già aveva attirato l'attenzione sull'importanza della trasmissione delle diatesi funzionali ${ }^{6}$, rilevò come $\mathbf{i}$ genetisti - i quali ammettono che cause esterne possano agire direttamente sul germe, ma non che modificazioni somatiche possano entrare a far parte del patrimonio ereditario - hanno trascurato di prendere in considerazione la possibilità che una variazione acquisita dal soma sotto l'influenza dell'ambiente provochi una modificazione corrispondente nel plasma germinale, la quale non corrisponda alla struttura e composizione del gene ${ }^{7}$.

Verrebbe così a determinarsi, fra plasma e gene, uno squilibrio il cui persistere potrebbe plausibilmente costringere il gene ad adattarsi al plasma, poichè sarebbe inconcepibile che, essendo l'adattamento la qualità caratteristica della vita, il gene debba ad essa sottrarsi completamente. In tal modo i caratteri acquisiti dal plasma sotto l'influenza diretta del soma ed indiretta dell'ambiente o del regime di vita entrerebbero a far parte del patrimonio ereditario.

Questa ipotesi, detta delle mutazioni adattative, può risultare difficile da verificare aggiungeva il Gini - poichè al sopravvenire di questo particolare tipo di mutazioni (criptomutazioni) non corrisponderebbe nessuna modificazione fenotipica; ma teoria ed esperienza concordano nel mostrare che gli organismi sono apparati che funzionano nell'interesse della specie, così che pare poco verosimile un'azione del soma tendente ad impedire il sorgere di mutazioni utili per la specie. E la teoria classica delle mutazioni porta proprio ad una tale conclusione.

7. L'ipotesi del Gini appare logicamente assai plausibile e con sempre maggiore frequenza vengono esposte vedute ad essa vicine, pur essendo evidenti i segni di riluttanza ad allontanarsi dai postulati della genetica classica.

Significativa al riguardo è la posizione assunta da Waddington in un recente lavoro sull'argomento ${ }^{8}$.

Egli si occupa, in particolare, dei fenomeni chiamati di adattamento pseudo-esogeno, cioè di "quei fenomeni che potrebbero essere spiegati facilmente come un effetto diretto di qualche fattore ambientale, se non fosse per il fatto indiscutibile che la caratteristica in questione $\dot{\mathrm{e}}$ in funzione del patrimonio ereditario e relativamente indipendente dall'ambiente".

5 A CARrel, Man, the unknown. Trad. it.: L'uomo, questo sconosciuto. Milano, 1936, p. 237.

${ }^{6}$ C. GINI, Le relazioni dell'eugenica con le altre scienze biologiche e sociali. "Atti del Primo Congresso Italiano di Eugenetica Sociale» (Milano, 20-23 settembre 1924). Roma, 1927, pp. 10-22 ed Eredità dei caratteri acquisiti o eredità della diatesi ? "Genus " 3,1-2 (1938), pp. 165-68.

" C. Gini, Mutazioni casuali o mutazioni adattative? "Genus », 4,3-4 (1940), pp. 81-82; vedi pure Teorie della popolazione. Roma, 1945, pp. 104-106

" C. H. WAdDington, L'evoluzione dell'adattamento all'ambiente. "Endeavour ", 12,47 (1953), pp. $134-39$. 
Già da questa definizione traspare in modo chiaro la tendenza a considerare in contrapposto adattamenti exatraorganici ed eredità genetica.

Tuttavia l'A. - di fronte alla domanda se sia possibile ritenere che le influenze ambientali abbiano una funzione del tutto secondaria nel determinare il fenomeno e che l'evoluzione di tale adattamento sia dovuta al processo selettivo delle mutazioni casuali che potrebbero avere prodotto un simile genotipo, anche qualora i fattori ambientali non avessero agito - dichiara assurdo il cercare una spiegazione per questi fenomeni senza considerare la possibile funzione dell'ambiente nella loro determinazione.

Di conseguenza Waddington espone una sua teoria dell'assimilazione genetica che può essere così riassunta.

La capacità di reagire agli stimoli esterni è dovuta ad un meccanismogenetico. La selezione naturale agisce con efficacia non solo a favore degli organismi che possiedono caratteristiche quasi ideali ma anche dei genotipi che tendono a produrre, in qualsiasi ambiente, fenotipi con siffatte caratteristiche; essa provoca, quindi, l'insorgenza di un meccanismo cibernetico per cui, ad un certo momento, lo stimolo iniziale rappresentato da fattori esterni diventa di poca importanza agli effetti dell'apparizione di certi caratteri durante lo sviluppo.

A questo punto potrebbe facilmente aver luogo una mutazione genica in grado di alterare una vicina parte dell'embrione, in modo tale da renderla capace di surrogare le funzioni dello stimolo esterno e di fissare genicamente la possibilità potenziale di sviluppare il carattere considerato.

Sembra prematuro formulare un giudizio sulla teoria esposta, la quale cerca di spiegare i fenomeni di adattamento pseudo-esogeno secondo la teoria genetica classica, in base alla selezione dei preesistenti geni condizionanti la capacità di reagire agli stimoli esterni ed alla stabilizzazione dei loro effetti, anche perchè i primi esperimenti compiuti dall'A. non hanno dato risultati univoci.

Da un punto di vista formale non si può nascondere una certa titubanza di fronte alla introduzione di termini come "caratteristiche quasi ideali » che andrebbero convenientemente definiti; inoltre non si vede come la selezione possa sistematicamente favorire i genotipi che tendono a produrre tali caratteristiche in qualsiasi ambiente: queste potrebbero benissimo essere, in certe condizioni, sfavorevoli alla conservazione dell'individuo e della specie, nel qual caso non si vede come non potrebbero soggiacere alla selezione.

8. Ma vi sono anche altri studi che recano direttamente elementi favorevoli alla teoria del Gini, illuminando pure i meccanismi attraverso i quali potrebbero verificarsi.

Particolarmente interessanti sembrano i risultati di ricerche sperimentali compiute da Demerec ${ }^{9}$ e che hanno condotto a formulare l'ipotesi che $\mathrm{i}$ mutageni non abbiano - come si riteneva - un'azione diretta sui geni, bensì un'azione indiretta attraverso il metabolismo cellulare. Ogni costituente del genoma mostrerebbe un tipo specifico di

- M. Demerec, Effetti genetici dei mutageni. Comunicazione presentata al «Congresso Internazionale di Genetica " tenuto a Bellagio nell'agosto 1953. 
reazione alle condizioni cellulari create dal mutageno, le quali potrebbero persistere, sia pure modificate da altri agenti, nelle cellule trattate fino al momento della loro divisione.

Ma non si possono trascurare gli studi sulla eredità citoplasmatica, riconosciuta da tempo ed alla quale solo abbastanza di recente $\mathrm{i}$ genetisti hanno rivolto l'attenzione che indubbiamente merita ${ }^{10}$.

Come è noto, le ricerche vengono compiute sul Paramecium aurelia, nel citoplasma del quale è stata accertata la presenza del cosiddetto fattore kappa determinante, unitamente al fattore $\mathrm{K}$ nucleare, la razza "killer "; tale fattore è stato denominato plasmagene e la sua natura non appare ancora chiara.

$\mathrm{Ma}$ vi è di più. Lo studio dell'ereditarietà dei tipi antigenici nel paramecio fa ritenere che il carattere del citoplasma - facilmente modificabile reversibilmente sotto l'influenza dell'ambiente - condizioni l'entrata in azione, cioè l'effetto sul fenotipo, dei geni presenti.

Il modello ereditario dell'antigene è stato anzi assunto a spiegare la differenziazione cellulare degli organismi pluricellulari: sarebbe lo stato del citoplasma - in parte controllato dall'ambiente - a determinare quali dei numerosi geni contenuti nella cellula regoleranno il corso degli eventi in quella cellula.

Che nel citoplasma esistano, pur sotto il controllo nucleare, entità capaci di autoriprodursi ed influenti sui processi di differenziazione, era stato del resto già messo in luce dalle ricerche di Lindegren e Billingham e Medawar.

9. In conclusione, sembra ormai certo che il plasma germinale svolge una parte non trascurabile nel quadro dei meccanismi ereditari. Risentendo esso delle modificazioni indotte dall'ambiente sul soma, è quindi molto plausibile ammettere col Gini la possibilità di mutazioni adatative, capaci di influire sulla evoluzione della specie.

Le considerazioni esposte nei paragrafi 4 e 5 lasciano però molto perplessi circa l'entità ed il carattere di una evoluzione avvenuta esclusivamente sotto l'influenza delle mutazioni adattative, delle mutazioni casuali e della selezione.

Tali perplessità conducono inevitabilmente ad ammettere - come è riconosciuto da diversi genetisti ${ }^{11}$ — che le tendenze evolutive, condizionate da forze esterne, siano guidate da forze interne, teoria che ha avuto in passato autorevoli esponenti in Naegeli, Eimer e Rosa e di cui il Gini mise in rilievo l'importanza da un punto di vista generale ${ }^{12}$, dopo averne sostenuto l'applicazione nel campo dei fenomeni demografici.

10 Vedi G. H. Beale, L'eredità nel paramecio. "Endeavour ", 13,49 (1954), pp. 33-36.

11 Vedi ad esempio C. JuCcI, Eredità ed evoluzione. "Genus », 1,1-2 (1934), pp. 22-27.

12 C. GiN1, Le relazioni dell'eugenica ecc., cit., pp. 13-14. 